\title{
GISAID: Global initiative on sharing all influenza data - from vision to reality
}

Yuelong Shu', John McCauley ${ }^{2}$

1. WHO Collaborating Center for Reference and Research on Influenza, Chinese National Influenza Center, National Institute for Viral Disease Control and Prevention, Chinese Center for Disease Control and Prevention, Beijing, China

2. WHO Collaborating Centre for Reference and Research on Influenza, Crick Worldwide Influenza Centre, the Francis Crick Institute, London, United Kingdom

Correspondence: John McCauley (John.McCauley@crick.ac.uk)

Citation style for this article:

Shu Y, McCauley J. GISAID: Global initiative on sharing all influenza data - from vision to reality. Euro Surveill. 2017;22(13):pii=30494. DOI: http://dx.doi. org/10.2807/1560-7917.ES.2017.22.13.30494

Article submitted on 27 March 2017 / accepted on 29 March 2017 / published on 30 March 2017

Ten years ago, a correspondence $[1,2]$, signed by more than 70 championed ' $A$ global initiative on sharing avian flu data' (GISAID) [3], leading to the GISAID Initiative in 2008. What started out as an expression of intent to foster international sharing of all influenza virus data and to publish results collaboratively has emerged as an indispensable mechanism for sharing influenza genetic sequence and metadata that embraces the interests and concerns of the wider influenza community, public health and animal health scientists, along with governments around the world. Today GISAID is recognised as an effective and trusted mechanism for rapid sharing of both published and 'unpublished' influenza data [4]. Its concept for incentivising data sharing established an alternative to data sharing via conventional public-domain archives.

In 2006, the reluctance of data sharing, in particular of avian $\mathrm{H}_{5} \mathrm{~N}_{1}$ influenza viruses, created an emergency bringing into focus certain limitations and inequities, such that the World Health Organization (WHO)'s Global Influenza Surveillance Network (now the Global Influenza Surveillance and Response System (GISRS) [5]) was criticised on several fronts, including limited global access to $\mathrm{H}_{5} \mathrm{~N}_{1}$ sequence data that were stored in a database hosted by the Los Alamos National Laboratories in the United States (US) $[6,7]$. This data repository, set up with financial support from the US Centers for Disease Control and Prevention (CDC) as a first attempt to share 'sensitive' data from affected countries, but was accessible only to those who were also providing $\mathrm{H}_{5} \mathrm{~N}_{1}$ sequence data. This limitedaccess approach restricted wider sharing of data prior to publication, which was vital for broader understanding of the progress of the emergent public and animal health threat. The need for greater transparency in data sharing and for acknowledgement of those contributing samples from $\mathrm{H}_{5} \mathrm{~N}_{1}$-infected patients and animals and related genetic sequence data was not satisfied by sharing data after formal publication via public-domain databases. Scientists charged with the day to day responsibilities of running WHO Collaborating Centres (CCs) for Influenza, National Influenza Centres and the World Organisation for Animal Health (OIE)/ Food and Agriculture Organization of the United Nations (FAO) [8] reference laboratories, were therefore eager to play a key role and provide scientific oversight in the creation and development of GISAID's data sharing platform that soon became essential for our work.

A unique collaboration ensued, involving, in addition to members of WHO's GISRS and OIE/FAO reference laboratories, the wider influenza research community along with officials in governmental institutions and non-governmental organisations. Facilitated by a wellconnected broadcast executive with background in licensing of intellectual property, an agreement was drawn up on the sharing of genetic data to meet emergency situations, without infringing intellectual property rights - the GISAID Database Access Agreement (DAA). The DAA governs each individual's access to and their use of data in GISAID's EpiFlu database [9]. It was this alliance between scientists and non-scientists, with a diversity of knowledge and experience, involved in drawing up an acceptable simple, yet enforceable, agreement which gained the trust and respect of the scientific community and public health and animal health authorities.

The essential features of the DAA encourage sharing of data by securing the provider's ownership of the data, requiring acknowledgement of those providing the samples and producing the data, while placing no restriction on the use of the data by registered users adhering to the DAA. It essentially defines a code of conduct between providers and users of data, cementing mutual respect for their respective complementary contributions, and upholding the collaborative ethos of WHO's GISRS, initially established 65 years ago this year [5]. 
Launched in 2008, the EpiFlu database was of key importance in the response to the 2009 influenza $\mathrm{A}\left(\mathrm{H}_{1} \mathrm{~N}_{1}\right)$ pandemic, allowing countries to readily follow the evolution of the new virus as it spread globally [10]. Acceptance of the GISAID sharing mechanism by providers and users of data, and the confidence of the influenza community, were further illustrated in 2013 by the unprecedented immediate release of the genetic sequences of Influenza $A\left(\mathrm{H}_{7} \mathrm{~N} 9\right)$ viruses from the first human cases, by Chinese scientists at the WHO Collaborating Centre for Influenza in Beijing $[11,12]$. Such events reaffirmed GISAID's applicability to timely sharing of crucial influenza data. The subsequent use of the sequence data to generate, develop and test candidate vaccine viruses by synthetic biology within a few weeks also demonstrated how GISAID successfully bridged this important 'technological' gap $[13,14]$. The paper by Bao et al. from Jiangsu province of China published in this issue once again confirms the importance of the timely sharing of data on the evolution of the $\mathrm{A}\left(\mathrm{H}_{7} \mathrm{~N} 9\right)$ viruses for global risk assessment. The authors analysed the recently isolated $\mathrm{H}_{7} \mathrm{~N} 9$ viruses form the fifth wave in Jiangsu province, and the results showed no significant viral mutations in key functional loci even though the $\mathrm{H}_{7} \mathrm{~N} 9$ viruses are under continuous dynamic reassortment and there is genetic heterogeneity. These findings should help to reduce concerns raised, even though the number of human infection with $\mathrm{H}_{7} \mathrm{~N}$ 9 virus increased sharply during the fifth wave in China.

GISAID provides the data-sharing platform particularly used by GISRS, through which sequence data considered by the WHO CCs in selecting viruses recommended for inclusion in seasonal and pre-pandemic vaccines are shared openly and on which research scientists, public and animal health officials and the pharmaceutical industry depend. Such openness of the most up-to-date data assists in an understanding of and enhances the credibility of the WHO recommendations for the composition of these seasonal and potential-pandemic vaccines.

Furthermore, in promoting the prompt sharing of data from potential pandemic zoonotic virus infections, as well as from seasonal influenza viruses, GISAID ensures a key tenet of the WHO Pandemic Influenza Preparedness (PIP) Framework [15], highlighting the critical role it plays in mounting an effective mitigating response. GISAID's ability to facilitate efficient global collaborations, such as the Global Consortium for $\mathrm{H}_{5} \mathrm{~N} 8$ and Related Influenza Viruses $[16,17]$, is central to monitoring phylogeographic interrelationships among, for example, $\mathrm{H}_{5}$ subtype viruses in wild and domestic birds in relation to their incidence, cross-border spread and veterinary impact, and assessing risk to animal and human health [18].

Traditional public-domain archives such as GenBank, where sharing and use of data takes place anonymously, fulfil a need for an archive of largely published data; however, that conventional method of data exchange notably has not been successful in encouraging rapid sharing of important data in epidemic or (potential) pandemic situations, such as those caused by Middle East respiratory syndrome coronavirus (MERS-CoV) and Ebola viruses. While the GISAID EpiFlu database is hosted and its sustainability ensured through the commitment of the Federal Republic of Germany [19], the establishment of GISAID and development of the EpiFlu database was reliant to a large extent on philanthropy of one individual and voluntary contributions and generosity of many others, together with some initial financial provision by the US CDC and the German Max Planck Society.

That GISAID has become accepted as a pragmatic means of meeting the needs of the influenza community in part reflects the particular characteristics of influenza and the continual need for year-round monitoring of the viruses circulating worldwide, essential for the biannual vaccine recommendations and assessment of the risk posed by frequent zoonotic infections by animal influenza viruses [20]. In the meantime, calls for an equivalent mechanism to promote the timely sharing of data in other urgent epidemic settings go largely unfulfilled $[21,22]$. A recent publication considered whether the 'paradigm shift' in data sharing by GISAID could be applied more generally to assist in preparedness for and response to other emergent infectious threats, such as those posed by Ebola virus [21] and Zika virus [23]. Such a trusted system could complement and take full advantage of the latest advances in rapid sequencing of specimens in the laboratory and in the field, for outbreak investigation [24].

Given the crucial importance of genetic data in improving our understanding of the progress of an emergent, potentially devastating epidemic, the effectiveness of GISAID in influenza pandemic preparedness is selfevident and provides important lessons for future pandemic threats. While the genetic makeup and the necessary associated data of the different viruses are distinct requiring separate databases/compartments for unambiguous analysis, the modi operandi for sharing genetic data are generic and the GISAID mechanism could be applied to other emerging pathogens. Indeed, the wider implementation of such a data sharing mechanism should be key in concerted efforts to contain spread of disease in animals and threats to human health, in realising the concept of One Health.

\section{Acknowledgements}

Peter Bogner, a philanthropist and broadcast executive with background in licensing of intellectual property and with a trust-based network of political contacts, acted as the principal instigator and active proponent and was instrumental in forging an alliance of all stakeholders leading to the creation of GISAID. Dr. Shu's research was supported by the National Key Re $\mathrm{R}_{-}$search and Development Program of China (2016YFC1200200) and the National Mega-projects for Infectious Diseases (2014ZX10004002).The work at the WHO 
CC in London was supported by the Francis Crick Institute which receives its core funding from Cancer Research UK (FC001030), the UK Medical Research Council (FC001030) and the Wellcome Trust (FCo01030).

\section{Conflict of interest}

None declared.

References

1. Boosting access to disease data. Nature. 2006;442(7106):957. DOI: 10.1038/442957a PMID: 16943803

2. Pearson H. Plan to pool bird-flu data takes off.Nature. 2006;442(7106):963. DOI: 10.1038/442963a PMID: 16943807

3. Bogner P, Capua I, Cox NJ, Lipman DJ, others. A global initiative on sharing avian flu data.Nature. 2006;442(7106):981. . Available from: http://www.nature.com/nature/journal/v442/ n7106/full/442981a.htmlDOI: 10.1038/442981a

4. Elbe S, Buckland-Merrett G. Data, disease and diplomacy GISAID's innovative contribution to global health.Global Challenges. 2017;1(1):33-46.

5. World Health Organization (WHO) Global Influenza Surveillance and Response System. (GISRS). Geneva: WHO; 2006. Available from: http://www.who.int/influenza/gisrs_laboratory/en/

6. Butler D. Flu researchers slam US agency for hoarding data. Nature. 2005;437(7058):458-9. DOI: 10.1038/437458a PMID: 16177749

7. Salzberg S. The contents of the syringe.Nature. 2008;454(7201):160-1. DOI: 10.1038/454160a PMID: 18615063

8. World Organisation for Animal Health (OIE)/Food and Agriculture Organization of the United Nations (FAO). Available from: http://www.oie.int/en/our-scientific-expertise/ reference-laboratories/list-of-laboratories/

9. The Global Initiative on Sharing All Influenza Data (GISAID). EpiFlu database access agreement (2008-2017). Available from: http://platform.gisaid.org/epi3/app_entities/entities/ downloads/epifludb_daa.pdf

10. Butler D. Swine flu goes global. Nature. 2009;458(7242):10823. DOI: $10.1038 / 4581082 a$ PMID: 19407756

11. Gao R, Cao B, Hu Y, Feng Z, Wang D, Hu W, et al. Human infection with a novel avian-origin influenza $A\left(\mathrm{H}_{7} \mathrm{~N} 9\right)$ virus. $\mathrm{N}$ Engl J Med. 2013;368(20):1888-97.

12. The fight against bird flu. Nature. 2013;496(7446):397. DOI: 10.1038/496397a PMID: 23627002

13. Vong S, O'Leary M, Feng Z. Early response to the emergence of influenza $A\left(\mathrm{H}_{7} \mathrm{~N} 9\right)$ virus in humans in China: the central role of prompt information sharing and public communication. Bull World Health Organ. 2014;92(4):303-8. DOI: 10.2471/ BLT.13.125989 PMID: 24700999

14. Dormitzer PR, Suphaphiphat P, Gibson DG, Wentworth DE, Stockwell TB, Algire MA, et al. Synthetic generation of influenza vaccine viruses for rapid response to pandemics. Sci Transl Med. 2013;5(185):185ra68. DOI: 10.1126/ scitranslmed.3006368 PMID: 23677594

15. Hekele A, Bertholet S, Archer J, Gibson DG, Palladino G, Brito

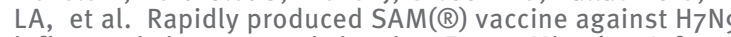
influenza is immunogenic in mice. Emerg Microbes Infect. 2013;2(8):e52. DOI: 10.1038/emi.2013.54 PMID: 26038486

16. Gostin LO, Phelan A, Stoto MA, Kraemer JD, Reddy KS. Virus sharing, genetic sequencing, and global health security. Science. 2014;345(6202):1295-6. DOI: 10.1126/science.1257622 PMID: 25214618

17. Global Consortium for $\mathrm{H}_{5} \mathrm{~N} 8$ and Related Influenza Viruses. Role for migratory wild birds in the global spread of avian influenza H5N8.Science. 2016;354(6309):213-7. DOI: 10.1126/ science.aaf8852 PMID: 27738169

18. Bi Y, Chen Q, Wang Q, Chen J, Jin T, Wong G, et al. Genesis, Evolution and Prevalence of $\mathrm{H}_{5} \mathrm{~N} 6$ Avian Influenza Viruses in China. Cell Host Microbe. 2016;20(6):810-21. DOI: 10.1016/j. chom.2016.10.022 PMID: 27916476

19. Federal Republic of Germany. Germany's statement on substantive issues and concerns regarding the pip framework and its implementation. Special Session of the PIP Advisory Group, 13 October 2015.Available from: http://www.who.int/ influenza/pip/advisory_group/german_statement_pipreview. pdf

20. Lipsitch M, Barclay W, Raman R, Russell CJ, Belser JA, Cobey $\mathrm{S}$, et al. Viral factors in influenza pandemic risk assessment. Elife. 2016;5:e18491. DOI: 10.7554/eLife.18491 PMID: 27834632
21. Yozwiak NL, Schaffner SF, Sabeti PC. Data sharing: Make outbreak research open access. Nature. 2015;518(7540):477-9. DOI: $10.1038 / 518477$ a PMID: 25719649

22. Modjarrad K, Moorthy VS, Millett P, Gsell PS, Roth C, Kieny MP. Developing Global Norms for Sharing Data and Results during Public Health Emergencies.PLoS Med. 2016;13(1):e1001935. DOI: 10.1371/journal.pmed.1001935 PMID: 26731342

23. Benefits of sharing. Nature. 2016;530(7589):129. DOI: 10.1038/530129a PMID: 26863943

24. Quick J, Loman NJ, Duraffour S, Simpson JT, Severi E, Cowley $\mathrm{L}$, et al. Real-time, portable genome sequencing for Ebola surveillance. Nature. 2016;530(7589):228-32. DOI: 10.1038/ nature16996 PMID: 26840485

\section{License and copyright}

This is an open-access article distributed under the terms of the Creative Commons Attribution (CC BY 4.0) Licence. You may share and adapt the material, but must give appropriate credit to the source, provide a link to the licence, and indicate if changes were made.

This article is copyright of the authors, 2017. 